\title{
The Association between Body Mass Index and Vitamin D Supplement Use among Adults in the United States
}

Carlos Orces ${ }^{1}$

1. Rheumatology, Laredo Medical Center, Laredo, USA

Corresponding author: Carlos Orces, corces07@yahoo.com

\section{Abstract \\ Background}

Obesity has been consistently associated with lower 25 -hydroxyvitamin D (25(OH)D) concentrations. BMI status also has a major impact on $25(\mathrm{OH}) \mathrm{D}$ levels among vitamin D supplement users. In our cross-sectional study, we aimed to discuss the prevalence of vitamin D supplement use and its effect on 25(OH)D levels according to BMI status among US adults.

\section{Methods}

The present analysis was based on data from the National Health and Nutrition Examination Survey (NHANES) cycles 2011-2012 through 2013-2014. The prevalence of vitamin D supplement use according to BMI status among subjects aged 20 years and older by selected characteristics was analyzed. Logistic regression models were assembled to examine the independent association of BMI status and vitamin D supplement use. Similarly, general linear models were used to assess the effect of daily vitamin D supplementation doses ( $<400 \mathrm{IU}, 400-800 \mathrm{IU}$, and $>800 \mathrm{IU}$ ) on $25(\mathrm{OH}) \mathrm{D}$ concentrations according to BMI status.

\section{Results}

Of 10,076 participants with a mean age of 47.2 [standard error (SE): 0.4 ] years, $57.5 \%$ (SE: 1.7) of normalweight and $63.5 \%$ (SE: 0.9) of obese subjects reported not taking vitamin D supplements over the previous 30 days. After adjustment for potential confounders, obese subjects were 1.3 times more likely to be nonusers of vitamin D supplements and were $24 \%$ less likely to take vitamin D supplements $\$ 400 \mathrm{IU} /$ day compared with their normal-weight counterparts. In addition, significant differences in mean 25(OH)D levels of vitamin D supplementation doses were consistently seen across BMI categories. Indeed, obese and overweight participants taking $>800 \mathrm{IU} /$ day of vitamin D supplements had 15.5 and $8.1 \mathrm{nmol} / \mathrm{L}$ lower mean concentrations of $25(\mathrm{OH}) \mathrm{D}$ than their normal-weight counterparts on average, respectively.

Received 08/12/2019

Review began 08/15/2019 Review ended 09/17/2019 Published 09/21/2019

\section{() Copyright 2019}

Orces. This is an open access article distributed under the terms of the Creative Commons Attribution License CC-BY 3.0., which permits unrestricted use, distribution, and reproduction in any medium, provided the original author and source are credited.

\section{Conclusion}

Obese subjects had a lower prevalence of vitamin D supplement use compared with their normal-weight counterparts. This nutritional disparity may also contribute to low 25(OH)D concentrations seen in obesity. However, a daily vitamin D supplement intake between 400 IU and 800 IU appears to be adequate among obese subjects to achieve optimal 25(OH) levels.

Categories: Endocrinology/Diabetes/Metabolism, Epidemiology/Public Health Keywords: obesity, vitamin d, supplements

\section{Introduction}

Lower serum 25-hydroxyvitamin D (25(OH)D) concentrations have been consistently reported across different latitudes in obese subjects compared with their normal-weight counterparts [1-9]. Similarly, interventional studies and randomized controlled trials (RCTs) of variable duration and vitamin D supplement doses have demonstrated an inverse 25(OH)D response according to BMI status and body fat [10-12]. Although the precise mechanism of low 25(OH)D levels in obese subjects has not been fully elucidated, previous studies have concluded that limited sun exposure, decreased bioavailability of vitamin $\mathrm{D}$ in fat tissue, or simply volumetric dilution may explain this association [4-6].

Notably, a recent analysis of the National Health and Nutrition Examination Survey (NHANES) demonstrated that obese older adults taking vitamin D supplements between 400 and 800 IU or >800 IU/day achieved 25(OH)D concentrations defined as sufficient [13]. However, Agarwal et al. described that more than $90 \%$ of US adults consumed less than the estimated average requirements of vitamin $\mathrm{D}$. In addition, dietary intake of vitamin D was significantly lower among the obese compared with their normal-weight 
counterparts. Agarwal et al. also observed a lower prevalence of vitamin D inadequacy among vitamin D supplement users compared to nonusers, which applies to obese subjects as well [14]. Despite this evidence, there are scarce population-based data regarding the relationship between BMI status and vitamin D supplement use. Our cross-sectional study aimed to address this and examine the prevalence of vitamin D supplement use and its effect on 25(OH)D levels according to BMI status in a nationally representative sample of adults.

\section{Materials And Methods}

NHANES is a biannual cross-sectional study conducted by the National Center for Health Statistics (NCHS) of the Centers for Disease Control and Prevention (CDC). The purpose of NHANES is to collect data about the health, nutritional status, and health behaviors of the noninstitutionalized civilian resident population of the US. The NHANES data were obtained using a complex, multistage probability sampling design to select a sample representative of the US civilian household population [15]. In this analysis, 11,329 participants aged 20 years and older were selected for the NHANES cycles 2011-2014. Those participants who had missing data on BMI $(n=572)$, vitamin D supplements $(n=214)$, or $25(O H) D$ levels $(n=1,002)$ were excluded, leaving a total study sample of 10,076 participants. Overall, subjects with missing data were more likely to be older, non-Hispanic blacks, with a college education, non-smokers, and physically inactive. They were mostly reported to be in good-to-excellent health.

\section{Characteristics of participants}

The demographics file provides individual, family, and household-level information on the following topics: the six-month time period when the examination was performed (November 1 through April 30 and May 1 through October 31), age, gender, race/ethnicity (Mexican-American and other Hispanic were grouped as Hispanic, non-Hispanic white, non-Hispanic black, and other race), education (<high school, high school/GED equivalent, some college or AA degree, and college graduate or above). The ratio of the familyincome-to-poverty threshold as a measure of socioeconomic status was calculated, and families with a ratio of $<1.00$ were considered below the poverty level. Participants were also asked the following query: "Is there a place that you usually go to when you are sick or need advice about your health?”. Those who responded affirmatively to this question were considered to have access to health care. Moreover, a physical-activity questionnaire was used to assess participants' leisure-time physical-activity status. The reported number of days and time in minutes spent performing vigorous or moderate leisure-time physical activity in the previous week were calculated. Based on the 2008 Physical Activity Guidelines for Americans, three levels of physical activity were created: 1 ) participants who engaged in $\geqslant 150 \mathrm{~min} /$ week of moderate activity, or $\geqslant 75$ $\mathrm{min} /$ week of vigorous activity, or $\geqslant 150 \mathrm{mins} /$ week of an equivalent combination were defined as physically active; 2 ) those who reported some physical activity but not enough to meet the active definition ( $>0$ to $<150$ $\mathrm{min} /$ week) were considered insufficiently active; 3 ) those who reported no physical activity were considered inactive [16].

In the mobile examination center, BMI was calculated as body weight $(\mathrm{kg})$ divided by height $\left(\mathrm{m}^{2}\right)$. Subjects were classified as normal-weight if they had a BMI of $<25.0 \mathrm{~kg} / \mathrm{m}^{2}$, overweight if the BMI was $25-29.9 \mathrm{~kg} / \mathrm{m}^{2}$, or obese in cases where BMI was $\geqslant 30 \mathrm{~kg} / \mathrm{m}^{2}$. Since underweight subjects accounted for a small number of participants $(n=171)$, they were grouped with the normal-weight participants. Participants also reported their smoking status and were classified as "current", "former, or "never" smokers. Self-reported general health condition was grouped as "good to excellent" or "fair to poor".

\section{Vitamin D supplement use}

The NHANES total dietary supplement file was used to examine the use of vitamin D supplements over the previous 30 days. All participants in the household sample were included in this file, including dietarysupplement users and non-users. Each total intake record contains the total number of supplements and antacids reported for that participant and the mean daily intake aggregates of 34 nutrients/dietary components from all supplements and antacids, as calculated using the NHANES dietary supplement database. Data were routinely examined for discrepancies and erroneous entries. Trained nutritionists reviewed incoming data and matched reported dietary-supplement entries to known supplements from the in-house product label database, where possible; sought additional product labels if feasible; assigned generic or default supplements as appropriate; transferred or removed products that were not considered dietary supplements; and assigned matching codes [17].

\section{5-hydroxyvitamin D concentrations}

The CDC-standardized liquid chromatography-tandem mass spectrometry (LC-MS/MS) method was used for measurement of 25(OH)D for NHANES 2011-2014, which allows laboratories and surveys to compare 25(OH)D measurements. CDC had decided to develop an LC-MS/MS method traceable to the NIST-reference materials for NHANES and had started using this method with NHANES 2007-2008. CDC recommends using the total 25(OH)D in SI units (nmol/L) measured directly by LC-MS/MS and converting this quantity to conventional units $(1 \mathrm{nmol} / \mathrm{L}=0.4066 \mathrm{ng} / \mathrm{mL})$ if needed. This method has better analytical specificity and sensitivity compared to immunoassay methods and fixed analytical goals for imprecision ( $\leqslant 10 \%)$ and bias 


\section{Cureus}

$(\leqslant 5 \%)[18]$

\section{Statistical analysis}

The descriptive characteristic of the study population stratified according to BMI status was reported as the percentage and mean values with their respective SEs. The chi-squared test was used to compare the proportion of participants taking vitamin D supplements stratified by BMI status across selected demographic, behavioral, and health characteristics. Subsequently, multiple logistic regression models were assembled to estimate the independent association of BMI status and vitamin D supplementation doses (none, $\geqslant 400 \mathrm{IU}$, and $\geqslant 800 \mathrm{IU}$ ) adjusted for six-month study period, age, gender, race/ethnicity, education, income, smoking habit, physical activity, health status, and access to health care. Similarly, in a subgroup analysis, adjusted general linear models were created to examine the effect of vitamin D supplement doses stratified according to BMI status on 25(OH)D concentrations. Statistical analyses were performed using SPSS Complex Sample software, V.17 (IBM, Armonk, New York) to incorporate constructed weights for the combined survey cycles and obtain unbiased, national estimates representative of the US population. A probability value $(\mathrm{P})$ of $<.05$ was considered statistically significant.

\section{Results}

The study sample comprised a total of 10,076 participants with a mean age of 47.2 (SE: 0.4 ) years. The table below (Table 1) shows the characteristics of participants stratified by BMI status. Overall, a higher proportion of participants were middle-aged, females, non-Hispanic whites, had less than a high-school education, were physically inactive, and reported good-to-excellent health.

Normal weight $(n=3,096), \%$ (SE)
Obese $(n=3,734), \%$ SE

Six-month period

Nov 1-Apr 30

$44.2(4.7)$

$47.4(4.6)$

May 1-Oct 31

$56.6(4.3)$

$55.8(4.7)$

$52.6(4.6)$

Age (years)

20-39

$45.7(2.0)$

32.7 (1.1)

$32.2(1.4)$

$40-59$

$31.3(1.6)$

39.2 (1.2)

$41.6(0.9)$

$\geq 60$

$23.0(1.4)$

$28.1(1.1)$

$26.2(0.9)$

Gender

Male

$43.0(0.9)$

$55.4(0.9)$

$45.9(0.9)$

Female

$57.0(0.9)$

$44.6(0.9)$

$54.1(0.9)$

Race/ethnicity

Hispanic

10.7 (1.3)

$15.2(1.8)$

$16.7(2.1)$

67.5 (2.3)

69.3 (2.5)

64.5 (3.0)

$8.7(1.2)$

$9.0(1.1)$

$14.5(1.9)$

$13.1(1.1)$

$6.6(0.6)$

$4.3(0.5)$

Education

Less than high school

High-school graduate

$24.0(2.1)$

26.0 (1.4)

29.3 (1.4)

$22.7(1.3)$

$25.7(1.1)$

$30.6(1.0)$

Some college or AA degree

$34.7(2.3)$

31.4 (1.5)

$28.8(1.0)$

College graduate or above

$18.6(2.4)$

16.9 (1.7)

$11.4(1.1)$

RIP

$<1.00$

$17.7(1.6)$

$14.1(1.0)$

$17.0(1.4)$

$\geq 1.00$

$82.3(1.6)$
$85.9(1.0)$

$83.0(1.4)$

Smoking status 


\section{Cureus}

\begin{tabular}{|llll} 
Never & $57.1(1.7)$ & $56.2(1.1)$ & $55.7(1.3)$ \\
Former & $19.0(1.1)$ & $26.4(1.1)$ & $26.4(1.2)$ \\
Current & $24.0(1.8)$ & $17.4(0.7)$ & $17.9(0.8)$ \\
Physical-activity status & & & $54.7(1.2)$ \\
Inactive & $37.7(1.9)$ & $43.5(1.4)$ & $17.3(0.8)$ \\
$<150$ min/week & $15.9(1.0)$ & $15.7(0.8)$ & $27.9(1.1)$ \\
$\geq 150$ min/week & $46.3(1.9)$ & $40.8(1.4)$ & $75.8(0.9)$ \\
General health condition & & & $24.2(0.9)$ \\
Good to excellent & $87.4(1.4)$ & $85.9(0.8)$ & $87.4(0.7)$ \\
Fair to poor & $12.6(1.4)$ & $14.1(0.8)$ & $12.6(0.7)$ \\
Place for health care & & &
\end{tabular}

TABLE 1: Characteristics of participants according to BMI categories, NHANES 2011-2014

SE: standard error; AA: associates of arts: RIP: ratio of family income to poverty

In general, higher 25(OH)D concentrations were consistently seen among older adults independently of their BMI status. In addition, 25(OH)D concentrations across age groups progressively decreased in overweight and obese subjects (Figure 1).

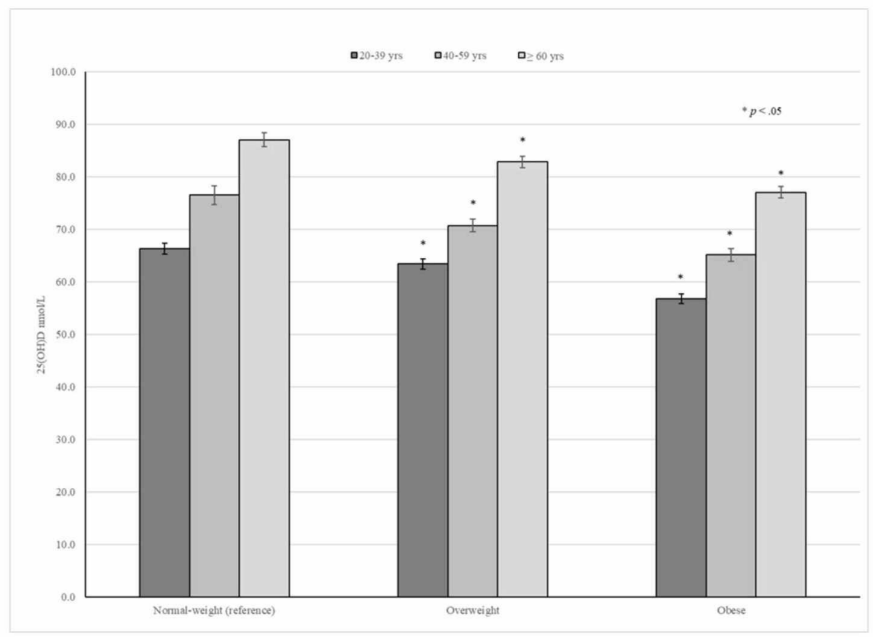

FIGURE 1: 25(OH)D concentrations according to age groups and BMI status

The table below (Table 2) shows the prevalence of vitamin D supplement use stratified by BMI status. Significantly, six-month time period during the fall and winter months, young and middle-aged subjects, women, non-Hispanic whites, household incomes above poverty level, physical activity, good-toexcellent health, and access to health care were characteristics associated significantly with a lower prevalence of vitamin D supplement use among obese subjects compared with their non-obese counterparts. 


\section{Cureus}

\begin{tabular}{|c|c|c|c|}
\hline & Normal weight $(n=3,096), \%$ (SE) & Overweight ( $n=3,246), \%$ (SE) & Obese ( $n=3,734), \%$ (SE) \\
\hline \multicolumn{4}{|l|}{ Six-month period } \\
\hline Nov 1-Apr 30 & $39.3(2.2)$ & $41.2(1.8)$ & $32.6(1.6)^{\star}$ \\
\hline May 1-Oct 31 & $45.1(2.3)$ & $45.2(1.8)$ & $40.0(1.1)$ \\
\hline \multicolumn{4}{|l|}{ Age (years) } \\
\hline 20-39 & $30.6(2.3)$ & $32.8(2.0)$ & $25.2(1.3)^{\star *}$ \\
\hline $40-59$ & $46.6(3.1)$ & $42.7(2.6)$ & $35.4(1.9)^{\star \star}$ \\
\hline$\geq 60$ & $60.8(2.6)$ & $56.6(2.6)$ & $52.3(2.6)$ \\
\hline \multicolumn{4}{|l|}{ Gender } \\
\hline Male & $32.1(1.6)$ & $38.5(1.5)$ & $31.2(1.4)^{\star}$ \\
\hline Female & $50.4(2.1)$ & $49.5(1.8)$ & $41.0(1.4)^{\star}$ \\
\hline \multicolumn{4}{|l|}{ Race/ethnicity } \\
\hline Hispanic & $24.4(2.6)$ & $23.5(1.9)$ & $24.2(1.8)$ \\
\hline Non-Hispanic white & $48.5(2.3)$ & $49.4(2.8)$ & $41.3(1.1)^{\star}$ \\
\hline Non-Hispanic black & $23.2(2.7)$ & $30.6(2.7)$ & $31.1(1.0)^{\star \star}$ \\
\hline Other races & $39.7(1.8)$ & $43.6(3.1)$ & $31.3(3.5)^{\star \star}$ \\
\hline \multicolumn{4}{|l|}{ Education } \\
\hline Less than high school & $29.6(1.7)$ & $33.0(2.5)$ & $29.7(1.9)$ \\
\hline High-school graduate & $33.0(2.3)$ & $40.4(2.5)$ & $36.7(1.5)$ \\
\hline Some college or AA degree & $51.5(3.1)$ & $45.9(1.8)$ & $40.2(2.1)^{\star \star}$ \\
\hline College graduate or above & $54.2(2.8)$ & $59.2(3.6)$ & $44.1(2.4)^{\star \star}$ \\
\hline \multicolumn{4}{|l|}{ RIP } \\
\hline$<1.00$ & $22.5(1.8)$ & $24.3(2.6)$ & $22.8(1.5)$ \\
\hline$\geq 1.00$ & $47.4(1.8)$ & $47.4(1.3)$ & $39.5(0.9)^{\star}$ \\
\hline \multicolumn{4}{|l|}{ Smoking status } \\
\hline Never & $43.9(2.1)$ & $45.0(1.9)$ & $37.8(1.4)^{\star \star}$ \\
\hline Former & $55.2(2.9)$ & $49.9(2.5)$ & $44.0(2.2)^{\star \star}$ \\
\hline Current & $29.3(2.8)$ & $28.2(2.3)$ & $21.5(1.6)^{\star \star}$ \\
\hline \multicolumn{4}{|l|}{ Physical-activity status } \\
\hline Inactive & $35.0(2.1)$ & $36.7(2.0)$ & $34.2(1.3)$ \\
\hline$<150 \mathrm{~min} /$ week & $48.1(2.6)$ & $46.3(1.8)$ & $41.7(2.4)$ \\
\hline$\geq 150 \mathrm{~mm} / \mathrm{week}$ & $46.9(2.2)$ & $49.4(2.1)$ & $38.0(1.8)^{*}$ \\
\hline \multicolumn{4}{|l|}{ General health condition } \\
\hline Good to excellent & $43.0(1.8)$ & 45.7 (1.4) & $36.9(0.9)^{\star}$ \\
\hline Fair to poor & $39.2(2.8)$ & $29.5(2.1)$ & $35.4(2.2)^{\star \star}$ \\
\hline \multicolumn{4}{|l|}{ Place for health care } \\
\hline Yes & $46.7(1.9)$ & $46.7(1.4)$ & $38.1(1.0)^{\star}$ \\
\hline No & $5.0(2.2)$ & $24.7(2.8)$ & $25.3(2.5)$ \\
\hline
\end{tabular}

TABLE 2: Prevalence of vitamin D supplement use according to BMI categories, NHANES 2011- 


\section{Cureus}

2014

SE: standard error; AA: associates of arts; RIP: ratio of family income to poverty

${ }^{*} \mathrm{P}:<0.001 ;{ }^{* *} \mathrm{P}:<0.05$

Overall, 1,903 (57.5\%), 1,989 (56.6\%), and 2,443 (63.5\%) of normal-weight, overweight, obese participants reported not taking vitamin D supplements over the previous 30 days, respectively. Moreover, only $28.1 \%$ of the obese subject reported taking vitamin D supplements $\geqslant 400 \mathrm{IU} /$ day. After adjustment for potential confounders, obese participants were 1.3 times more likely to be nonusers of vitamin D supplements and were $24 \%$ less likely to use vitamin D supplements $\geqslant 400 \mathrm{IU} /$ day compared with their normal-weight counterparts. Although obese participants were less likely to take vitamin D supplements $\geqslant 800 \mathrm{IU} /$ day, differences across BMI categories were not statistically significant (Table 3).

\begin{tabular}{|c|c|c|c|}
\hline & Normal weight $(n=3,096)$ & Overweight $(n=3,246)$ & Obese $(n=3,734)$ \\
\hline \multicolumn{4}{|l|}{ No vitamin D supplement } \\
\hline$\%$ (SE) & $57.5(1.7)$ & $56.6(1.3)$ & $63.5(0.9)^{\star}$ \\
\hline Crude OR (95\% Cl) & 1.00 (ref) & $0.96(0.82,1.13)$ & $1.28(1.10,1.50)$ \\
\hline Adjusted OR $(95 \% \mathrm{Cl})^{\mathrm{a}}$ & 1.00 (ref) & $0.99(0.84,1.7)$ & $1.30(1.10,1.54)$ \\
\hline \multicolumn{4}{|l|}{ Vitamin $D \geq 400 \mathrm{IU} /$ day } \\
\hline$\%$ (SE) & $32.9(1.5)$ & $32.7(1.3)$ & $28.1(0.9)^{\star}$ \\
\hline Crude OR (95\% Cl) & 1.00 (ref) & $0.99(0.84,1.15)$ & $0.79(0.68,0.91)$ \\
\hline Adjusted OR $(95 \% \mathrm{Cl})^{\mathrm{a}}$ & 1.00 (ref) & $0.93(0.79,1.09)$ & $0.76(0.64,0.91)$ \\
\hline \multicolumn{4}{|l|}{ Vitamin $D \geq 800$ IU/day } \\
\hline$\%$ (SE) & $18.8(1.5)$ & $18.5(1.1)$ & $16.4(0.8)$ \\
\hline OR (95\% Cl) & 1.00 (ref) & $0.98(0.82,1.16)$ & $0.84(0.69,1.03)$ \\
\hline Adjusted OR $(95 \% \mathrm{Cl})^{\mathrm{a}}$ & 1.00 (ref) & $1.01(0.81,1.26)$ & $0.86(0.65,1.13)$ \\
\hline
\end{tabular}

\section{TABLE 3: Association of BMI status and vitamin D supplement use}

OR: odds ratio; $\mathrm{Cl}$ : confidence interval

aModels adjusted for the six-month study period, age, gender, race-ethnicity, education, income, smoking status, physical activity, health status, and access to health care

${ }^{*} \mathrm{p}:<0.05$

Notably, mean 25(OH)D levels progressively increased as vitamin D supplementation doses increased. However, significant differences in mean $25(\mathrm{OH}) \mathrm{D}$ levels according to vitamin D supplementation doses were consistently seen across BMI categories. In fact, obese and overweight participants taking vitamin D supplements >800 IU/day had 15.5 and $8.1 \mathrm{nmol} / \mathrm{L}$ lower mean concentrations of 25(OH)D than their normalweight counterparts on average, respectively (Figure 2 ). 


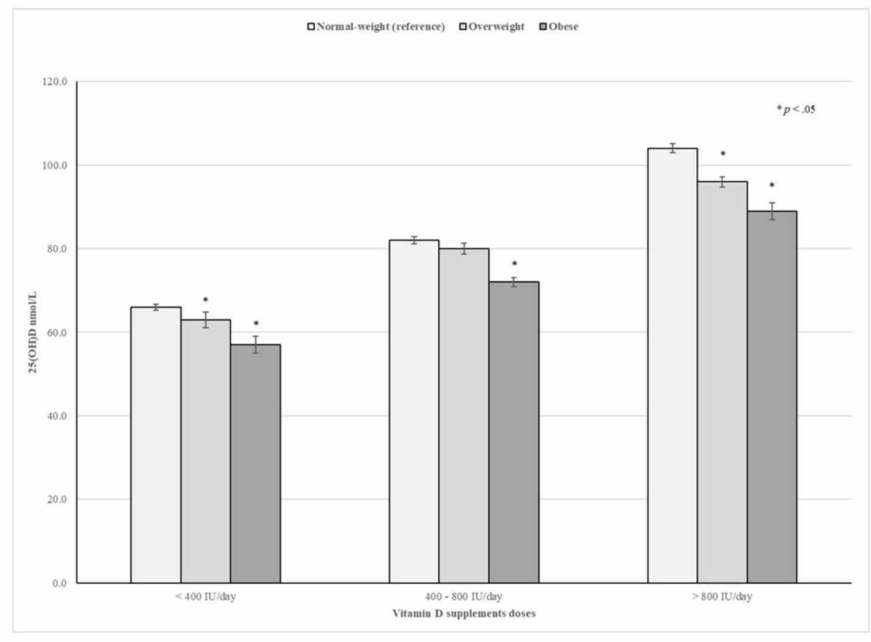

\section{FIGURE 2: The effect of vitamin D supplements on 25(OH)D concentrations according to BMI status}

\section{Discussion}

In this nationally representative sample of adults, vitamin D supplement use significantly differed according to BMI status. Overall, the use of vitamin D supplements was consistently lower among obese subjects compared with their normal-weight counterparts. This nutritional disparity was particularly seen among young and middle-aged participants, females, non-Hispanic blacks, subjects with a college education, and those with access to health care. Previous studies have also reported a higher risk of inadequate intake for several key micronutrients and dietary quality among obese adults compared to adults with a healthy weight [14,19-20]. Notably, the present findings indicate that only one-third of US adults met the 2011 Institute of Medicine (IOM) estimated average requirement for vitamin D intake ( $\geqslant 400 \mathrm{IU} /$ day) from dietary supplements during the study period [21]. Moreover, after adjustment for potential confounders, obese subjects were $24 \%$ less likely to report taking vitamin D supplements $\$ 400$ IU/day than their normal-weight counterparts. Recently, Blumberg et al. reported that among US adults, the use of multi-vitamin at any frequency significantly increased nutrient intakes and decreased the percentage of the population with inadequate intakes for most micronutrients, as compared with food alone [22]. Blumberg et al. also observed that the prevalence of vitamin D intake inadequacy decreased from $92.5 \%$ with food-only to $17.3 \%$ when vitamin D supplements were added. In addition, as compared with nonusers of vitamin D supplements, participants who reported taking vitamin D supplements 21 or more days decreased the odds of having vitamin $\mathrm{D}$ deficiency by $76 \%[22]$.

Several studies have shown that BMI is a major determinant of $25(\mathrm{OH}) \mathrm{D}$ concentrations following vitamin $\mathrm{D}$ supplementation [11-12,23-24]. In fact, a placebo-controlled trial of vitamin D supplementation conducted among postmenopausal white women with vitamin $\mathrm{D}$ insufficiency demonstrated that overweight and obese women had lower levels of $25(\mathrm{OH}) \mathrm{D}$ concentration (about $12.5 \mathrm{nmol} / \mathrm{L}$ and $17.5 \mathrm{nmol} / \mathrm{L}$, respectively) compared with women with normal-weight at 12 months regardless of whether the vitamin D dose was administered or not [24]. Likewise, a large study among healthy volunteers in Alberta, Canada, reported that $600 \mathrm{IU} /$ day of vitamin D supplementation achieved an average $25(\mathrm{OH}) \mathrm{D}$ concentration of 83, 76 and, $66 \mathrm{nmol} / \mathrm{L}$ in normal-weight, overweight, and obese participants, respectively [25]. The present results also demonstrate that $25(\mathrm{OH}) \mathrm{D}$ concentrations differed according to BMI status among vitamin $\mathrm{D}$ supplement users. Indeed, even after adjustment for potential confounders, normal-weight, overweight, and obese participants who reported taking vitamin D supplements between 400 and 800IU/day had mean $25(\mathrm{OH}) \mathrm{D}$ concentrations at 82,80 , and $72 \mathrm{nmol} / \mathrm{L}$, respectively.

Obese subjects who use vitamin D supplements between 400 and 800IU/day or higher doses had mean $25(\mathrm{OH}) \mathrm{D}$ concentrations consistently higher than $50 \mathrm{nmol} / \mathrm{L}$, which is the $25(\mathrm{OH}) \mathrm{D}$ cutoff level defined as sufficient by the IOM report [21]. Although, the Endocrine Society has recommended that obese subjects may need at least two or three more vitamin D intakes (at least 6,000 to 10,000 IU/day) to treat or prevent vitamin $\mathrm{D}$ deficiency, the present findings demonstrate that obese subjects who reported taking vitamin $\mathrm{D}$ supplements between 400 and $800 \mathrm{IU} /$ day were able to reach optimal 25(OH)D concentrations $(\geqslant 50$ $\mathrm{nmol} / \mathrm{L}$ ). Therefore, the IOM-recommended vitamin D dietary allowance of $600 \mathrm{IU} /$ day for subjects aged 2070 years and $800 \mathrm{IU} /$ day for those $>70$ years should be considered adequate for obese adults until further research evidence is available. 
concentrations have consistently reported parallel curves across BMI status, suggesting that the differences in $25(\mathrm{OH}) \mathrm{D}$ concentrations are most likely due to a volume dilution effect, which is consistent with the present findings [5,11,24]. Moreover, Gallagher et al. reported a small difference in dose-response curves of vitamin D supplements on 25(OH)D levels between normal-weight and obese subjects, suggesting that inadequate intake of vitamin D may also account for lower 25(OH)D levels seen in obesity [11].

Interestingly, young adults had a lower prevalence of vitamin D supplement use compared with their middle-aged and older adult counterparts, which was also more marked in obese participants. Similarly, young adults had lower 25(OH)D concentrations across BMI status compared with those in other age groups. In fact, the lowest $25(\mathrm{OH}) \mathrm{D}$ concentration at $56.8 \mathrm{nmol} / \mathrm{L}$ was seen among obese adults aged 20-39 years. Thus, these findings suggest that obese young adults might be at increased risk of vitamin D inadequacy and should be counseled about vitamin D supplementation. Although the effect of low 25(OH)D concentrations among obese subjects on bone mineralization is not very clear, a recent nationwide crosssectional analysis demonstrated that BMI was positively associated with femoral-neck-bone mineral density (BMD) among adults aged 50 years and older, irrespective of their sex or race [26]. In contrast, a prospective study conducted among participants in the Health ABC study reported that obese older adults had significantly lower mean femoral-neck BMD than those with normal weight at 10 years of follow-up [27].

Several limitations should be considered while interpreting the study results. First, because of the NHANES cross-sectional design, the present findings do not necessarily infer causation. Second, sun exposure and sun-protective behavior variables, which may affect the synthesis of $25(\mathrm{OH}) \mathrm{D} 3$, were not explored in the present analysis. Third, the effect of latitude on participants' 25(OH)D concentrations was unknown. Fourth, most sociodemographic variables were self-reported, which may have led to recall bias. Finally, although there are few naturally occurring food sources of vitamin D, dietary vitamin D was not examined in this study. However, dietary vitamin D may also contribute to 25(OH)D concentrations [21].

\section{Conclusions}

Our study concluded that obese subjects had a lower prevalence of vitamin D supplement use compared with their normal-weight counterparts. This nutritional disparity may also contribute to low $25(\mathrm{OH}) \mathrm{D}$ concentrations seen in obesity. However, a daily vitamin D supplement intake between $400 \mathrm{IU}$ and $800 \mathrm{IU}$ appears to be adequate among obese subjects to achieve optimal $25(\mathrm{OH})$ levels.

\section{Additional Information \\ Disclosures}

Human subjects: Consent was obtained by all participants in this study. Animal subjects: All authors have confirmed that this study did not involve animal subjects or tissue. Conflicts of interest: In compliance with the ICMJE uniform disclosure form, all authors declare the following: Payment/services info: All authors have declared that no financial support was received from any organization for the submitted work. Financial relationships: All authors have declared that they have no financial relationships at present or within the previous three years with any organizations that might have an interest in the submitted work. Other relationships: All authors have declared that there are no other relationships or activities that could appear to have influenced the submitted work.

\section{References}

1. Carrelli A, Bucovsky M, Horst R, et al.: Vitamin D storage in adipose tissue of obese and normal weight women. J Bone Miner Res. 2017, 32:237-42. 10.1002/jbmr.2979

2. Palacios C, Gil K, Pérez CM, Joshipura K: Determinants of vitamin D status among overweight and obese Puerto Rican adults. Ann Nutr Metab. 2012, 60:35-43. 10.1159/000335282

3. Liel Y, Ulmer E, Shary J, Hollis BW, Bell NH: Low circulating vitamin D in obesity . Calcif Tissue Int. 1988, 43:199-201. 10.1007/bf02555135

4. Wortsman J, Matsuoka LY, Chen TC, Lu Z, Holick MF: Decreased bioavailability of vitamin D in obesity . Am J Clin Nutr. 2000, 72:690-3. 10.1093/ajcn/72.3.690

5. Drincic AT, Armas LA, Van Diest EE, Heaney RP: Volumetric dilution, rather than sequestration best explains the low vitamin D status of obesity. Obesity (Silver Spring). 2012, 20:1444-8. 10.1038/oby.2011.404

6. Savastano S, Barrea L, Savanelli MC, Nappi F, Di Somma C, Orio F, Colao A: Low vitamin D status and obesity: role of nutritionist. Rev Endocr Metab Disord. 2017, 18:215-25. 10.1007/s11154-017-9410-7

7. Samuel L, Borrell LN: The effect of body mass index on optimal vitamin D status in U.S. adults: The National Health and Nutrition Examination Survey 2001-2006. Ann Epidemiol. 2013, 23:409-14. 10.1016/j.annepidem.2013.05.011

8. Arunabh S, Pollack S, Yeh J, Aloia JF: Body fat content and 25-hydroxyvitamin D levels in healthy women. J Clin Endocrinol Metab. 2003, 88:157-61. 10.1210/jc.2002-020978

9. Orces $\mathrm{CH}$ : The association between obesity and vitamin D status among older adults in Ecuador: analysis of the SABE survey. Nutr Hosp. 2018, 35:1066-71. 10.20960/nh.1752

10. Camozzi V, Frigo AC, Zaninotto M, et al.: 25-Hydroxycholecalciferol response to single oral cholecalciferol loading in the normal weight, overweight, and obese. Osteoporos Int. 2016, 27:2593-602. 10.1007/s00198016-3574-y

11. Gallagher JC, Yalamanchili V, Smith LM: The effect of vitamin D supplementation on serum 25(OH)D in thin 
and obese women. J Steroid Biochem Mol Biol. 2013, 136:195-200. 10.1016/j.jsbmb.2012.12.003

12. Blum M, Dallal GE, Dawson-Hughes B: Body size and serum 25 hydroxy vitamin D response to oral supplements in healthy older adults. J Am Coll Nutr. 2008, 27:274-9. 10.1080/07315724.2008.10719700

13. Orces C, Lorenzo C, Guarneros JE: The Prevalence and Determinants of Vitamin D Inadequacy among U.S. Older Adults: National Health and Nutrition Examination Survey 2007-2014. Cureus. 2019, Accessed: September 21, 2019: https://www.cureus.com/articles/21039-the-prevalence-and-determinants-of-vitamind-inadequacy-among-us-older-adults-n.... 10.7759/cureus.5300

14. Agarwal S, Reider C, Brooks JR, Fulgoni VL 3rd: Comparison of prevalence of inadequate nutrient intake based on body weight status of adults in the United States: an analysis of NHANES 2001-2008. J Am Coll Nutr. 2015, 34:126-34. 10.1080/07315724.2014.901196

15. NHANES survey methods and analytic guidelines. (2019). Accessed: May 2, 2019: https://wwwn.cdc.gov/nchs/nhanes/analyticguidelines.aspx.

16. 2008 physical activity guidelines for Americans. (2008). Accessed: September 21, 2019: https://health.gov/paguidelines/2008/.

17. National Health and Nutrition Examination Survey; 2013-2014 data documentation, codebook, and frequencies. (2016). Accessed: May 2, 2019: https://wwwn.cdc.gov/Nchs/Nhanes/20132014/DSQTOT_H.htm.

18. Analytical note for 25-Hydroxyvitamin D data analysis using NHANES III (1988-1994), NHANES 2001-2006, and NHANES 2007-2010 (October 2015). Accessed: July 9, 2019: https://wwwn.cdc.gov/Nchs/Nhanes/VitaminD/AnalyticalNote.aspx.

19. Damms-Machado A, Weser G, Bischoff SC: Micronutrient deficiency in obese subjects undergoing low calorie diet. Nutr J. 2012, 11:34. Accessed: September 21, 2019: 10.1186/1475-2891-11-34

20. Tidwell DK, Valliant MW: Higher amounts of body fat are associated with inadequate intakes of calcium and vitamin D in African American women. Nutr Res. 2011, 31:527-36. 10.1016/j.nutres.2011.06.005

21. Dietary Reference Intakes for Calcium and Vitamin D . Ross AC, Taylor CL, Yaktine AL, Del Valle HB (ed): National Academies Press, Washington, DC; 2011.

22. Blumberg JB, Frei BB, Fulgoni VL, Weaver CM, Zeisel SH: Impact of frequency of multi-vitamin/multimineral supplement intake on nutritional adequacy and nutrient deficiencies in U.S. adults. Nutrients. 2017, 9:E849. Accessed: September 21, 2019: 10.3390/nu9080849

23. Tepper S, Shahar DR, Geva D, Ish-Shalom S: Predictors of serum 25(OH)D increase following bimonthly supplementation with 100,000IU vitamin D in healthy, men aged 25-65 years. J Steroid Biochem Mol Biol. 2014, 144:163-6. 10.1016/j.jsbmb.2013.12.005

24. Gallagher JC, Sai A, Templin T 2nd, Smith L: Dose response to vitamin D supplementation in postmenopausal women: a randomized trial. Ann Intern Med. 2012, 156:425-37. 10.7326/0003-4819-156-6201203200-00005

25. Ekwaru JP, Zwicker JD, Holick MF, Giovannucci E, Veugelers PJ: The importance of body weight for the dose response relationship of oral vitamin D supplementation and serum 25-hydroxyvitamin D in healthy volunteers. PLoS One. 2014, 9:e111265. Accessed: September 21, 2019: 10.1371/journal.pone.0111265

26. Lloyd JT, Alley DE, Hawkes WG, Hochberg MC, Waldstein SR, Orwig DL: Body mass index is positively associated with bone mineral density in US older adults. Arch Osteoporos. 2014, 9:175. Accessed: September 21, 2019: 10.1007/s11657-014-0175-2

27. Lloyd JT, Alley DE, Hochberg MC, et al.: ABC Study. Changes in bone mineral density over time by body mass index in the Health ABC study. Osteoporos Int. 2016, 27:2109-16. 10.1007/s00198-016-3506-X 